\title{
Neonatal form of biotin-thiamine-responsive basal ganglia disease. Clues to diagnosis
}

\author{
Aydan Değerliyurt ${ }^{1}$, Mehmet Gündüz ${ }^{2}$, Serdar Ceylaner $^{3}$, Özlem Ünal ${ }^{2}$, Sevim Ünal ${ }^{4}$ \\ Departments of ${ }^{1}$ Pediatric Neurology, ${ }^{2}$ Pediatric Metabolism and Nutrition, and ${ }^{4}$ Neonatology, Ankara Pediatrics, Hematology- \\ Oncology Training and Research Hospital; ${ }^{3}$ Intergen Genetics Centre, Ankara, Turkey. E- mail: degerliyurta@gmail.com \\ Received: 27th January 2018, Revised: 19th March 2018, Accepted: 20th March 2018
}

SUMMARY: Değerliyurt A, Gündüz M, Ceylaner S, Ünal Ö, Ünal S. Neonatal form of biotin-thiamine-responsive basal ganglia disease. Clues to diagnosis. Turk J Pediatr 2019; 61: 261-266.

Biotin-thiamine-responsive basal ganglia disease is characterized by seizures, dystonia and encephalopathy attacks, with an acute-subacute onset in childhood. It causes cerebral damage especially with caudate head and putamen involvement and may lead to severe sequelae and even death if left untreated. We report a patient with the neonatal form of biotin-thiamine-responsive basal ganglia disease who presented with encephalopathy and lactic acidosis in the neonatal period together with the diagnostic magnetic resonance imaging (MRI) clues. MRI in the neonatal period revealed bilateral involvement of the putamen, thalamus, and perirolandic cortical regions. However, MRI obtained at 32 months revealed involvement of the caudate nuclei in addition to the putamen and thalami. The neuroimaging findings of our patient and relevant literature indicate that patients with biotin-thiamine-responsive basal ganglia disease who are symptomatic in the neonatal period have putamen, thalami, and perirolandic cortical involvement. However, these patients do not have caudate involvement, unlike the patients who present in childhood.

Key words: basal ganglia, biotin-thiamine-responsive basal ganglia disease, Leigh syndrome, SLC19A3, thiamine transporter 2.

Biotin-thiamine-responsive basal ganglia disease (BTBGD) is characterized by seizures, dystonia and encephalopathy attacks with an acutesubacute onset in childhood. The patients respond to early biotin-thiamine treatment. It can however result in death if left untreated. ${ }^{1}$ The cause is mutations in the SLC19A3 gene leading to deterioration of thiamine transport in neurons via thiamine transporter 2 (hTHTR2). ${ }^{2,3}$ SLC19A3 gene mutations lead to 4 different clinical pictures based on the age of onset: 1. Leigh syndrome-like phenotype characterized by acute encephalopathy and lactic acidosis in the neonatal period. ${ }^{4-7}$ 2. A severe disease occurring in early infancy characterized by epileptic spasms, severe psychomotor retardation, progressive brain atrophy and bilateral thalamus and basal ganglia lesions. ${ }^{8} 3$. Biotin-thiamine-responsive basal ganglia disease in childhood. ${ }^{9}$ 4. Wernicke's encephalopathy-like condition in the second decade of life. ${ }^{10}$
Elevated lactate concentrations are found in body fluids in most neonatal and early infantile patients in contrast to childhood forms. Patients with more widespread encephalopathy generally start in the earlier infantile period and have a more severe phenotype. ${ }^{11}$ However, there is probably a clinical continuum among patients. ${ }^{12}$ A good differential diagnosis and early diagnosis are very important as starting appropriate treatment is of vital importance. We present a case of the neonatal form of BTBGD together with the diagnostic magnetic resonance imaging (MRI) findings.

\section{Case Report}

A 28-day-old male infant presented to our hospital with inconsolable crying, upward deviation of the eyes, opisthotonus, and seizures. There was nothing of significance in the prenatal and natal history of the patient. The parents were first-degree relatives. A sister 


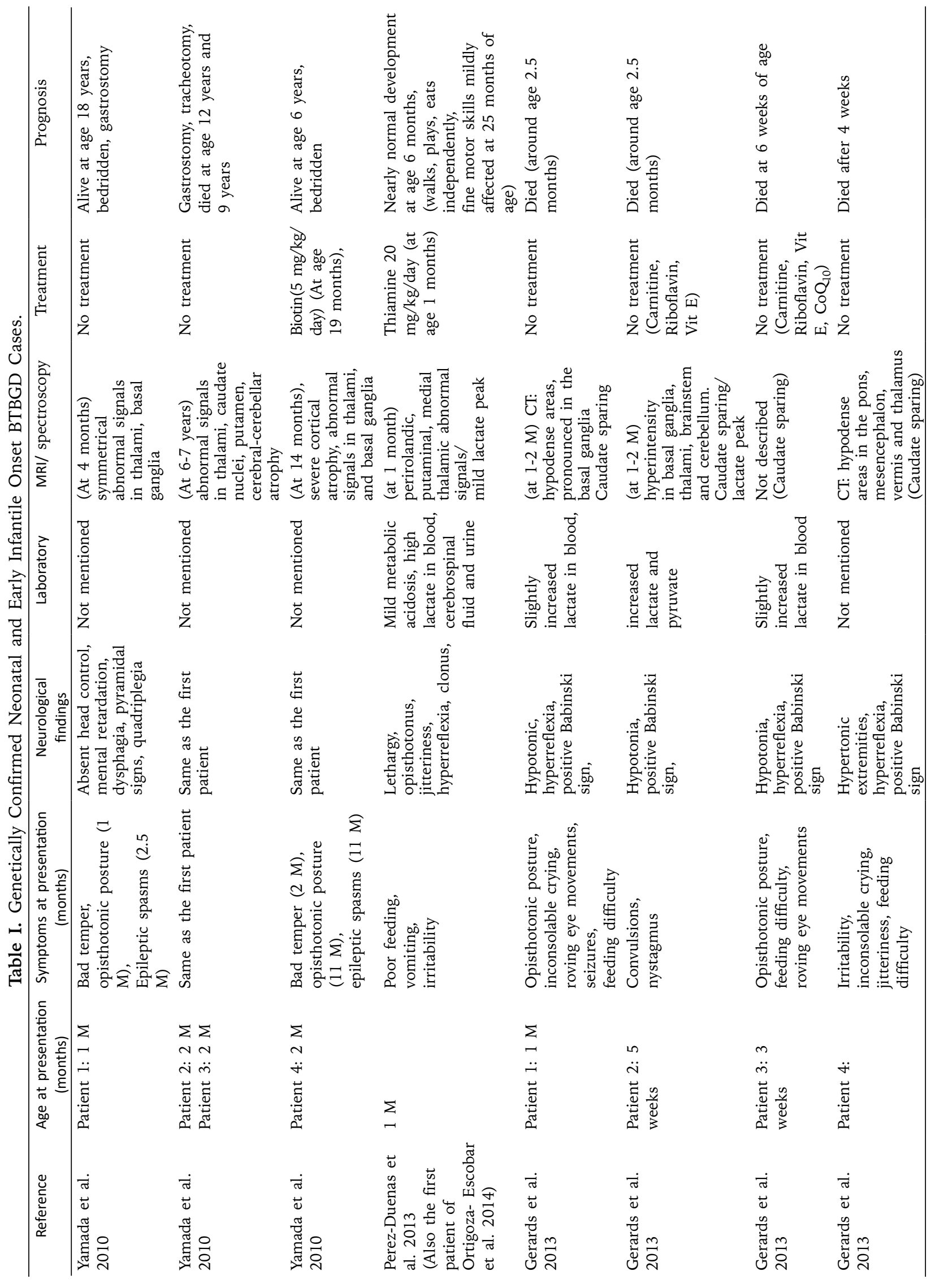



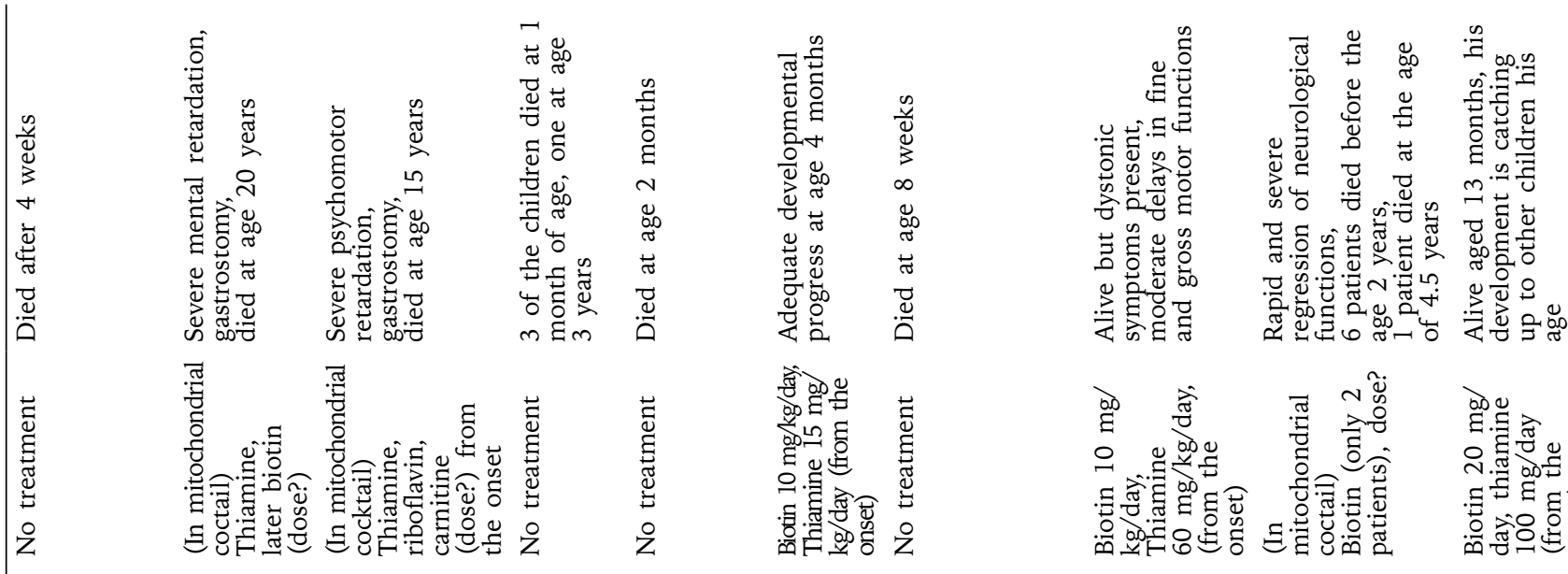

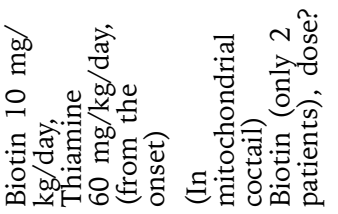

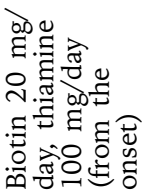

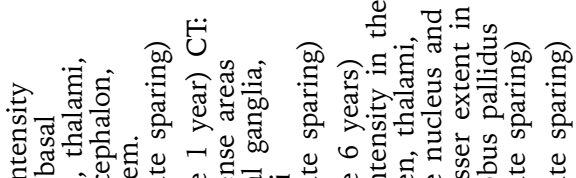

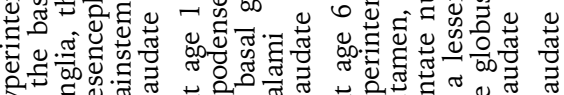

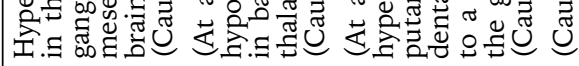

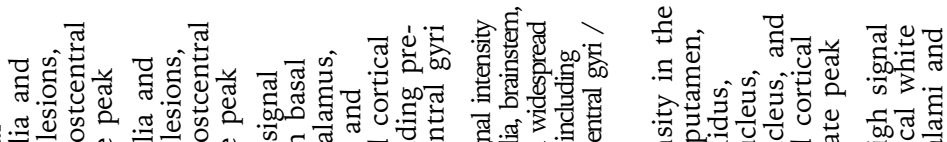

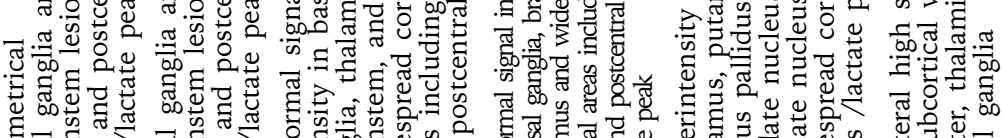

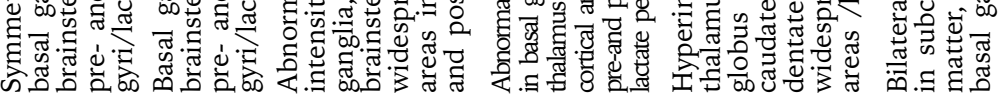

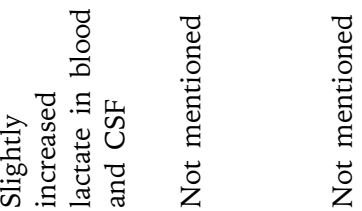

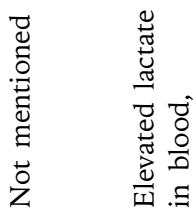

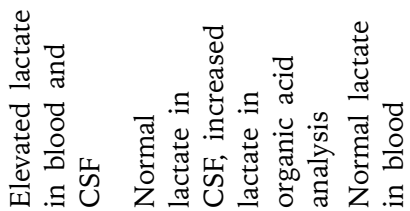

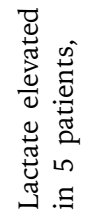

\section{童

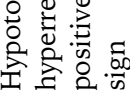

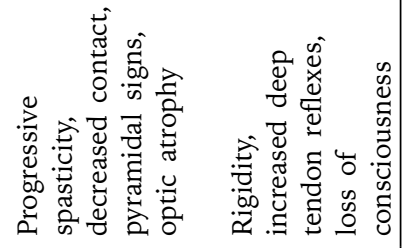

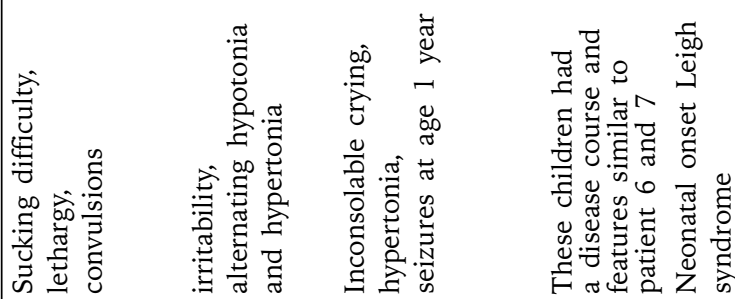

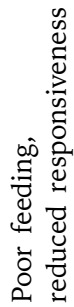

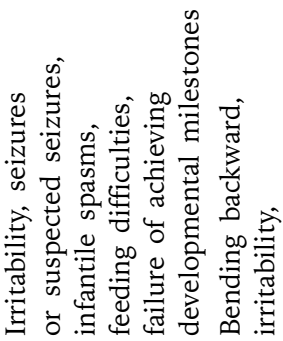

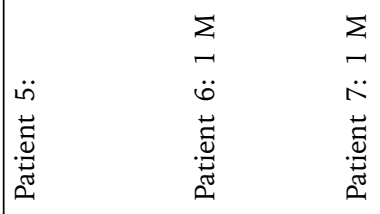

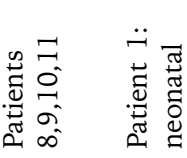

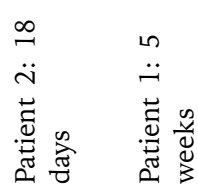

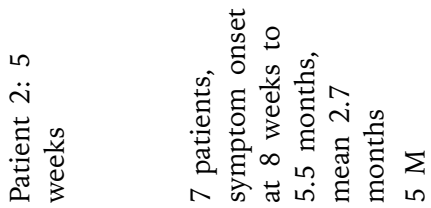

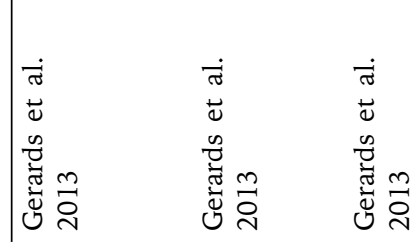

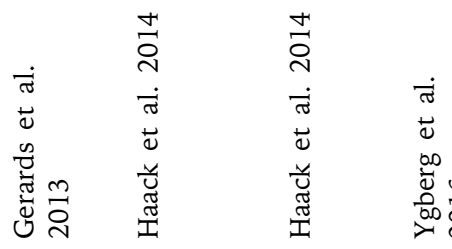

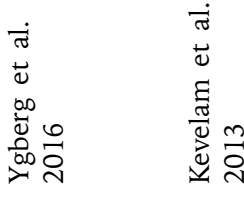

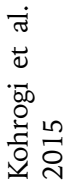




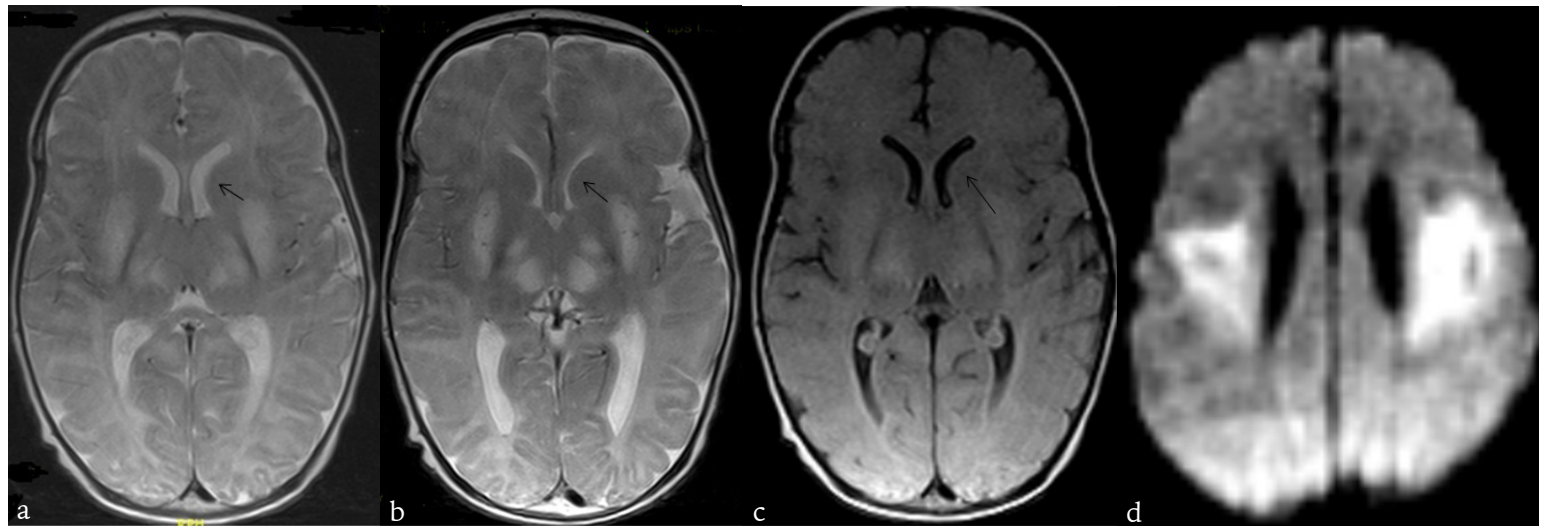

Fig. 1. Cerebral MRI showed edema and signal increase in bilateral putamen, globus pallidus, thalamus at (a-b) axial T2 images, (c) FLAIR images, (d) perirolandic area with diffusion restriction at age 1 month. Caudate nucleus heads were spared (arrow)

of the patient had died at the age of 2 months with a diagnosis of Leigh syndrome. The patient's cerebrospinal fluid (CSF) biochemistry was normal. There was no growth on culture. The blood lactate level was $51 \mathrm{mg} / \mathrm{dl}$ (normal $<19.8 \mathrm{mg} / \mathrm{dl}$ ) and the pyruvate level was 1.04 $\mathrm{mg} / \mathrm{dl}$ (normal $<0.7 \mathrm{mg} / \mathrm{dl}$ ). The lactate to pyruvate ratio was markedly increased. Detailed metabolic studies (ammonia level, blood gases, blood and CSF amino acids, urine organic acids, acylcarnitines, biotinidase activity) revealed normal results. Cerebral MRI showed edema and signal increase in T2 and FLAIR images at bilateral putamen, globus pallidus, thalamus and pons dorsal segments and the perirolandic area together with diffusion restriction (Fig. 1). The patient was considered to be suffering from Leigh syndrome and was started carnitine, coenzyme $\mathrm{Q}_{10}$, and $\mathrm{B}$ group vitamins (half tablet daily of Benexol ${ }^{\circledR}$ containing $250 \mathrm{mg}$ thiamine $\mathrm{HCl})$. The seizures resolved completely, and the

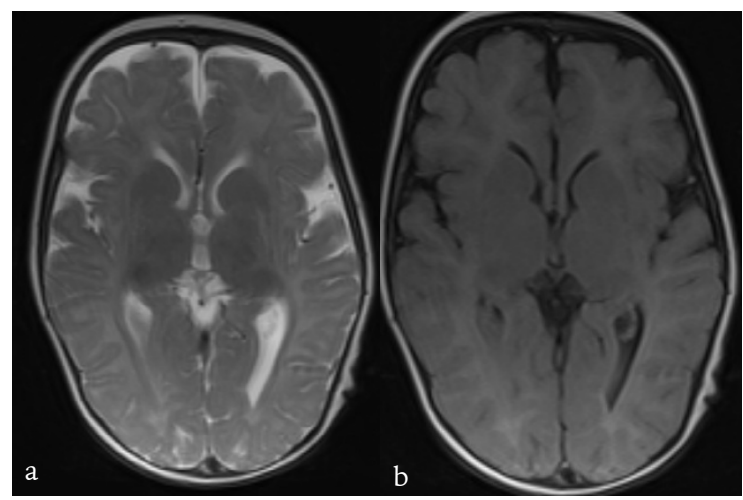

Fig. 2. Cerebral MRI revealed disappearance of abnormal signals at (a) axial T2, (b) FLAIR images at age 6 months. patient was discharged on phenobarbital and phenytoin treatments. No seizures occurred until the patient was 5 months old when jerks and flexor spasms started and hypsarrhythmia was detected on electroencephalography (EEG). The MRI showed that the signal changes and diffusion restriction in the initial MRI had disappeared when the patient was 6 months old (Fig. 2). MR spectroscopy at the age of 1 year revealed increased lactate peak.

Immunohistochemical analysis of the muscle biopsy at 2 years of age was normal. The patient eventually developed microcephaly, hypotonia, motor retardation and intermittent dystonia during follow-up. MRI at the age of 32 months revealed signal changes at bilateral caudate heads and putamen extending to the thalami and ventral globus pallidus together with cerebral atrophy (Fig. 3), leading to a suspicion of biotin-thiamine-responsive basal ganglia disease. After obtaining parental written informed consent, we planned to perform SLC19A3 gene sequence analysis but could not manage amplification with any one of the amplicons using in-house designed primers. We then decided to perform deletion analysis with positive and negative control samples, which revealed a homozygote deletion of the complete gene. As this is a very severe mutation, this abnormality causes complete absence and loss of function of the gene products.

The patient used the recommended vitamin treatment irregularly until the time of diagnosis. Thiamine $30 \mathrm{mg} / \mathrm{kg} /$ day and biotin $10 \mathrm{mg} / \mathrm{kg} /$ day were started at the age of 3 years. 


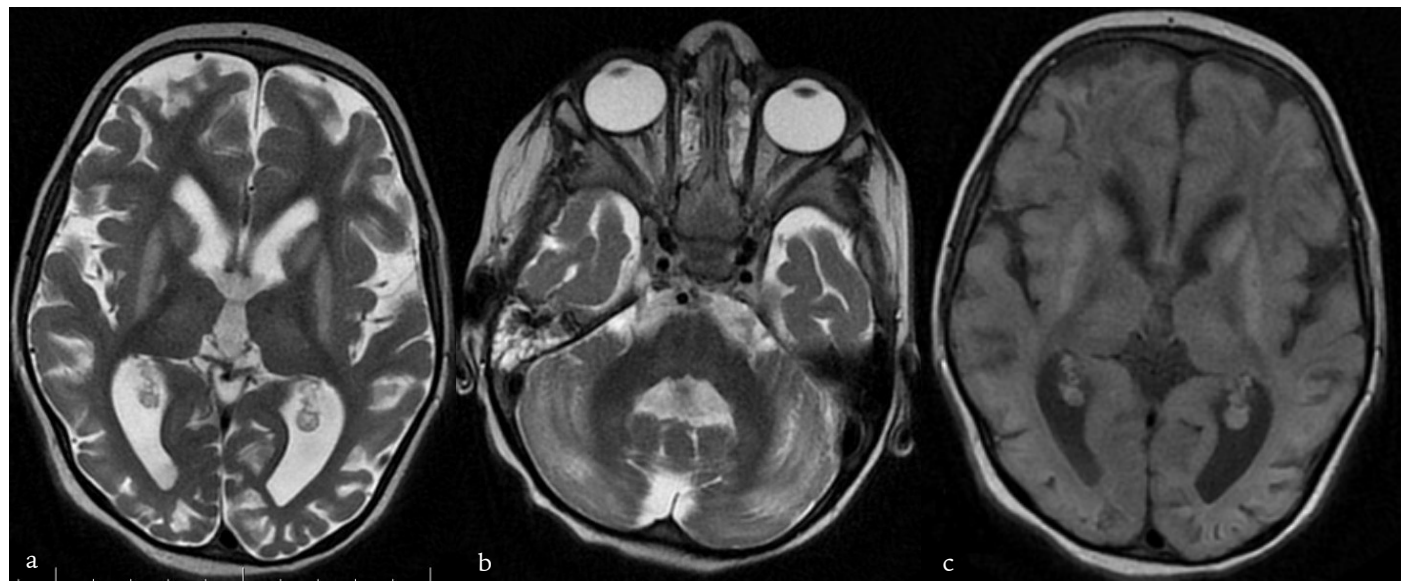

Fig. 3. (a-b) Axial T2 images, (c) FLAIR image at the age of 32 months revealed hyperintense signal changes in bilateral caudate nucleus heads and putamen extending to the thalami and ventral globus pallidus together with widespread cerebral atrophy.

The patient is currently 5 years old. Head control is present, but he cannot sit unsupported. He recognizes family members, can smile, understands some simple commands, and can speak a few words. However, he cannot play with his toys and the intermittent dystonia continues. Deep tendon reflexes are bright, Babinski reflex is bilaterally positive and extremity spasticity is present together with axial hypotonia. He suffers one or two seizures daily and is therefore on triple antiepileptic treatment.

\section{Discussion}

Early diagnosis of BTBGD is vital importance as only 6 of the 29 reported patients who became symptomatic in the newborn period or early infancy ( $<6$ months) are still alive (Table I). Swelling and vasogenic edema in the putamen and bilateral caudate nucleus heads are typical on MRI. Signal changes can also be seen in the mesencephalon, cortical-subcortical regions, and thalamic nuclei while the globus pallidus is usually protected in the acute stage of the childhood form. ${ }^{1,13}$ Our patient did not show caudate involvement on the early MRI in neonatal period unlike childhood onset patients.

Some studies have also emphasized that involvement of the basal ganglia, thalami, brain stem, cerebellum, and perirolandic cortical area were present but the caudate nuclei were preserved on MRIs in 12 newborns. ${ }^{4,5,12}$ Similar results were also reported in the acute MRIs of 4 patients with the neonatal Leigh phenotype in two other studies. No information on caudate involvement was provided in either study but the caudate nuclei are seen to be preserved in the figures. ${ }^{6,7}$ Another 2 studies have reported involvement of the basal nuclei including the caudates, thalami, white matter, cortex, pons and midbrain. However, these patients had presented in early infancy and not in the neonatal period. 8,11

These results indicate that those who are symptomatic in the neonatal period do not have caudate involvement, unlike patients presenting in later periods. ${ }^{4-7}$ However, these patients can show caudate involvement after a while, as also seen in our case. The different age-related energy requirements of the various brain regions or the differing localization of gene expression by age could lead to the lack of involvement from the disorder in the caudate nuclei in the neonatal period. ${ }^{4}$

Another feature of our patient was the involvement of the perirolandic cortical areas in the acute stage. Cortical involvement in the acute stage has been reported in newborns with detailed MRI reports. Basal ganglia involvement accompanying cortical involvement is a well-known feature of the childhood form of BTBGD. ${ }^{9}$

A few patients who became symptomatic in the newborn period or early infancy ( $<6$ months) were alive up to the time of reporting. Four of the surviving 6 patients were diagnosed and treated early. $4,6,7,12,14$ Our patient was only diagnosed at 3 years and not during the 
newborn period when he first presented but he had received irregular thiamine and biotin within the mitochondrial cocktail up to the time of diagnosis. Perhaps this is the reason for survival despite severe sequelae. Recognizing the MRI findings of the neonatal form is therefore of great importance in making the diagnosis early and starting treatment before sequelae develop.

One of the major causes of lactic acidosis and encephalopathy in the newborn is Leigh syndrome due to pyruvate dehydrogenase complex deficiency. However, the lactate/ pyruvate ratio is normal and neuroimaging frequently shows globus pallidus involvement and structural brain abnormalities in pyruvate dehydrogenase complex deficiency. ${ }^{12,15}$ We therefore did not consider pyruvate dehydrogenase complex deficiency in our patient. Leigh syndrome shows pronounced putamen involvement together with caudate nuclei and brainstem involvement on MRI. Basal ganglia and cortical involvement together is not typical of Leigh syndrome. ${ }^{16}$ Moreover, thalamic lesions were found at a low rate in Leigh syndrome. ${ }^{17}$ The presence of marked thalamic involvement in newborns with the SLC19A3 mutation is another important finding against Leigh syndrome (Table I).

In conclusion, one must consider biotinthiamine-responsive basal ganglia disease, one of the rare Leigh syndrome phenotypes that can be treated, if putamen, thalamic and cortical involvement is present while the caudate nuclei are preserved on MRI in patients who present with lactic acidosis and encephalopathy in the neonatal period. Treatment should be started promptly to ensure a better prognosis and avoid the development of sequelae and death.

\section{REFERENCES}

1. Alfadhel M, Almuntashri M, Jadah RH, et al. Biotinresponsive basal ganglia disease should be renamed biotin-thiamine-responsive basal ganglia disease: a retrospective review of the clinical, radiological and molecular findings of 18 new cases. Orphanet J Rare Dis 2013; 8: 83.

2. Zeng WQ, Al-Yamani E, Acierno JS Jr, et al. Biotinresponsive basal ganglia disease maps to $2 \mathrm{q} 36.3$ and is due to mutations in SLC19A3. Am J Hum Genet 2005; 77 : 16-26.
3. Subramanian VS, Marchant JS, Said HM. Biotinresponsive basal ganglia disease-linked mutations inhibit thiamine transport via hTHTR2: biotin is not a substrate for hTHTR2. Am J Physiol Cell Physiol 2006; 291; C851-C859.

4. Pérez-Dueñas B, Serrano M, Rebollo M, et al. Reversible lactic acidosis in a newborn with thiamine transporter-2 deficiency. Pediatrics 2013; 131: e1670-e1675.

5. Gerards M, Kamps R, van Oevelen J, et al. Exome sequencing reveals a novel Moroccan founder mutation in SLC19A3 as a new cause of early-childhood fatal Leigh syndrome. Brain 2013; 136 (Pt3): 882-890.

6. Haack TB, Klee D, Strom TM, et al. Infantile Leigh-like syndrome caused by SLC19A3 mutations is a treatable disease. Brain 2014; 137 (Pt 9) : e295.

7. Ygberg S, Naess K, Eriksson M, et al. Biotin and thiamine responsive basal ganglia disease: $\mathrm{A}$ vital differential diagnosis in infants with severe encephalopathy. Eur J Paediatr Neurol 2016; 20: 457461.

8. Yamada K, Miura K, Hara K, et al. A wide spectrum of clinical and brain MRI findings in patients with SLC19A3 mutations. BMC Med Genet 2010; 11: 171.

9. Tabarki B, Al-Shafi S, Al-Shahwan S, et al. Biotinresponsive basal ganglia disease revisited. Clinical radiologic, and genetic findings. Neurology 2013; 80: 261-267.

10. Kono S, Miyajima H, Yoshida K, Togawa A, Shirakawa $\mathrm{K}$, Suzuki H. Mutations in a thiamine-transporter gene and Wernicke's-like encephalopathy. N Engl J Med 2009; 360: 1792-1794.

11. Kevelam SH, Bugiani M, Salomons GS, et al. Exome sequencing reveals mutated SLC19A3 in patients with an early-infantile, lethal encephalopathy. Brain 2013; 136 (Pt 5): 1534-1543.

12. Ortigoza-Escobar JD, Serrano M, Molero M, et al. Thiamine transporter-2 deficiency: outcome and treatment monitoring. Orphanet J Rare Dis 2014; 9: 92.

13. Kassem H, Wafaie A, Alsuhibani S, Farid T. Biotinresponsive basal ganglia disease: neuroimaging features before and after treatment. Am J Neuroradiol 2014; 35: 1990-1995.

14. Kohrogi K, Imagawa E, Muto Y, et al. Biotin-responsive basal ganglia disease: a case diagnosed by whole exome sequencing. J Hum Genet 2015; 60: 381-385.

15. Bhandary S, Aguan K. Pyruvate dehydrogenase complex deficiency and its relationship with epilepsy frequency - An overview. Epilepsy Res 2015; 116: 40-52.

16. Distelmaier F, Huppke P, Pieperhoff P, et al. Biotinresponsive basal ganglia disease: a treatable differential diagnosis of Leigh syndrome. JIMD Rep 2014; 13: 53-57.

17. Bonfante E, Koenig MK, Adejumo RB, Perinjelil V, Riascos RF. The neuroimaging of Leigh syndrome: case series and review of the literature. Pediatr Radiol 2016; 46: 443-451. 